\title{
Peptide nanofibers for controlled growth factor release
}

\author{
"Due to the critical modulatory role of growth factors in development of and regeneration of tissue, controlled delivery of \\ growth factors to the defect site is crucial for regenerative medicine studies."
}

Keywords: growth factor delivery $\backsim$ peptide $\backsim$ peptide nanofibers

Growth factors are soluble proteins that are capable of inducing cellular responses including cell proliferation, survival, migration and differentiation. They function through binding to specific receptors on the surface of target cells and inducing signaling cascade in the cytoplasm. Depending on the proximity of the target cells, growth factors can be classified asparacrine (target cell is nearby), autocrine (target cell is the same cell that secreted the growth factor), juxtracrine (target cell must be in physical contact with growth factor-receptor complex) or intracrine (growth factor-receptor complex is internalized).

The ability of the growth factors to induce cellular response primarily depends on target cell type, cell number, type and number of receptors on target cells and finally the intracellular signal transduction cascades following growth factor binding to its receptor. In addition to these parameters, cellular response also depends on the ability of the growth factor to diffuse through the extracellular matrix (ECM). A growth factor can induce various cellular responses depending on the receptor and target cell type. The same receptor can conduct distinct messages depending on the intracellular transduction pathways, which can differ from one cell type to another [1]. In the vicinity of cells, growth factors are often bound to proteoglycan molecules and this interaction modulates growth factor action and potency by regulating spatial presentation, diffusion properties, synthesis and degradation. For example, latent TGF $\beta$-binding protein is an ECM protein that stores TGF $\beta$, where TGF $\beta$ can be released upon ECM remodeling by serum proteases [2].

Due to the critical modulatory role of growth factors in development and regeneration of tissue, controlled delivery of growth factors to the defect site is crucial for regenerative medicine studies. Peptide nanofibers provide synthetic platforms to reconstruct both morphological characteristics and bioactive properties of cellular microenvironments. Geometry of the high-aspect-ratio nanofibers formed by peptides allows dense presentation of bioactive epitopes to cells [3-5]. Due to these characteristics, peptide nanofibers are widely used as growth factor carriers and delivery vehicles in regenerative medicine studies.

\section{Physical encapsulation of growth factors}

Growth factors can be encapsulated in peptide nanostructures through noncovalent interactions such as electrostatic interactions [6]. Release of encapsulated growth factors into surrounding tissue can be controlled through porosity, density and degradation rate of peptide nanofiber network. Synthetic systems inspired by natural ECM features can provide more controlled propagation of growth factor signals to regulate cell decisions. Incorporation of growth factor-binding sites similar to ones in the ECM into synthetic biomaterials has attracted wide attention as a promising strategy to localize growth factor action on target cells or tissues. For example, heparin-mimetic systems have been used as delivery platforms to increase growth factor affinity to receptor for sustained release of growth factors and for extended half-life because heparin aids binding of growth factors to receptors more efficiently. Heparin-mimetic peptide nanofiber system was designed to mimic heparan sulfate, and bind to VEGF [7]. When endothelial cells were cultured on the heparin-mimetic peptide nanofibers, angiogenesis was induced in vitro without addition of exogenous growth factors and fast neo-vascularization was observed in rat cornea. These peptide nanofibers also showed specific binding affinity to other heparin-binding growth factors such as HGF and FGF-2 [8].

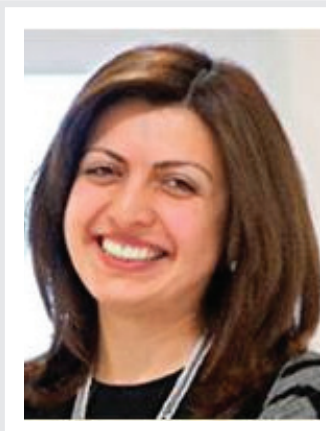

Ayse B Tekinay

Author for correspondence: Institute of Materials Science \& Nanotechnology, National Nanotechnology Research Center, Bilkent University, Ankara,

Turkey 06800

Tel.: +90 3122903572

E-mail: atekinay@

unam.bilkent.edu.tr

Mustafa $\mathbf{O}$ Guler

Institute of Materials Science \& Nanotechnology, National Nanotechnology Research Center, Bilkent University, Ankara, Turkey 06800

\section{Didem Mumcuoglu}

Institute of Materials Science \& Nanotechnology, National Nanotechnology Research Center, Bilkent University, Ankara, Turkey 06800

Seher Ustun

Institute of Materials Science \& Nanotechnology, National Nanotechnology Research Center, Bilkent University, Ankara,

Turkey 06800 
In addition, both heparin-mimetic and lamininmimetic peptide nanostructures were used to induce neural differentiation through binding to NGF inducing neurogenesis. This system was shown to be effective in neural differentiation even in the presence of axonal growth inhibitor chondroitin sulfate proteoglycans [9]. Heparin-binding peptide amphiphiles (HBPA) were also inspired by growth factor and heparin interactions. Cardin-Weintraub heparin-binding peptide domain was used to functionalize peptide nanofibers to specifically bind to heparan sulfate. In the presence of sulfated glycosaminoglycans, these peptide nanofibers captured heparin-binding growth factors, demonstrated prolonged release of angiogenic growth factors, and promoted vascularization when injected into rat cornea [10]. Effect of HBPA molecules on angiogenesis was also tested in a membrane system that was formed by HBPA, heparin and hyaluronic acid. This system was shown to promote retention of angiogenic factors and increase angiogenesis in chicken chorioallantoic membrane assay [11]. Heparin-binding domain was also exploited in a RADA16 system by coupling the heparin-binding domain, LRKKLGKA, to the $\mathrm{C}$ terminus of RADA16 self-assembling peptide molecules [12-13].

\section{"Delivery systems must induce cells with the accurate concentration of growth factors at the precise time frame to prevent undesired cellular decisions."}

\section{Growth factor tethering systems}

Immobilization of growth factors by covalent binding to delivery material provides extended bioavailability, slower degradation, less internalization by cells, and spatial control of growth factor. However, covalent binding of growth factors to self-assembling peptides is not favored since growth factor binding may impede with self-assembly process. Previously, avidin-bound peptide amphiphile molecules were demonstrated for potential proteinligand binding [14]. In addition, researchers conjugated biotin molecules at the C-termini of peptide molecules, where biotinylated IGF-1 was complexed with streptavidin and coupled to biotinylated self-assembling peptides. This sandwich strategy did not hinder self-assembly process and allow prolonged IGF-1 delivery and activity [15].

\section{Stimuli-responsive approaches}

Stimuli-responsive materials are based on rapid transition of material upon stimulus such as $\mathrm{pH}$ or temperature change, or drug or enzyme concentration. Growth factor delivery requires precise adjustment of the release rate because differentiation should be induced in a timely manner. For example, timing VEGF release to initiate blood vessel formation and PDGF to stabilize already formed vessels is critical in angiogenesis. Otherwise an initial bolus delivery of PDGF may result in destabilization of vessels followed by regression. Protease-dependent release systems release growth factors from delivery systems as material is degraded upon cleavage by proteases [16]. MMP enzymes are ECM-remodeling enzymes found in ECM in a tissue-specific manner. Peptides including cleavage sites for specific MMPs can be degraded by cell-secreted MMPs. In one study, peptide nanofibers functionalized with MMP-13 cleavage site showed sequence specific degradation when hydrogel was implanted into tissue [17]. Incorporation of MMP-binding site into peptide nanofibers provide tissue- and cell-dependent degradation and in turn controlled bioavailability of growth factors. In another approach, assembly and disassembly processes of self-assembling peptides were controlled through incorporation of phosphorylation sites recognized by PKA. When peptide nanofibers were treated with PKA, they were phosphorylated, which resulted in disassembly of peptide nanostructures and release of encapsulated bioactive reagents [18].

\section{Growth factor mimicking strategies}

Since growth factors have potency to induce several different cellular pathways in different tissues, mode of growth factor delivery must target the specific cell population and minimize transmission of signal to other cells or tissue. Delivery systems must induce cells with the accurate concentration of growth factors at the precise time frame to prevent undesired cellular decisions. In general, growth factors loaded in delivery systems are used at high concentrations to achieve sustained and slow-release of growth factors to target tissue for longer durations. These delivery strategies rely on use of recombinant exogenous growth factors, which may cause immunogenic responses and limit the clinical applicability. Growth factor-mimetic or -capturing systems have been used to eliminate these problems. Self-assembled peptide nanofibers presenting a VEGF-mimicking peptide sequence efficiently 
induced tissue recovery in a hind-limb ischemia model of cardiovascular disease [19]. As discussed above, heparin mimetic strategies were also used as endogenously-released growth factor capturing systems [7]. In addition, researchers designed peptide molecules that display TGF $\beta$-binding epitopes to sequester endogenously released TGF $\beta$ growth factor. TGF $\beta$-binding PA significantly enhanced recovery of microfracturetreated cartilage defects without the addition of exogenous growth factors when implanted with mesenchymal stem cells [20].

\section{“...peptide nanofibers enhanced cell differentiation and survival without exogenous growth factor use by capturing endogenous growth factor or by mimicking growth factor itself."}

\section{Conclusions}

During development and regeneration of tissues, ECM modulates cellular functions through modulating concentration, transmission and localization of bioactive molecules including growth factors. In this sense, growth factor delivery systems inspired by native ECM should enhance growth factor potency and action by regulating localization, presentation and concentration of growth factors. Self-assembling peptides can be modified at single amino acid level to incorporate bioactive signals to better mimic ECM-counterparts. Up-to-date, controlled and slow release of growth factors from self-assembled peptide constructs was achieved using noncovalent interactions and $3 \mathrm{D}$ porous environment and it is possible to tune growth factor release by including glycosaminoglycans or glycosaminoglycans-mimetic molecules. However, application of these growth factor delivery systems in clinical cases will be limited, since they rely on exogenous recombinant growth factors. This challenge may be bypassed by using bioactive epitopes that capture and present endogenous growth factors or by using growth factor-mimics. Several studies showed that engineered peptide nanofibers enhanced cell differentiation and survival without exogenous growth factor use by capturing endogenous growth factors or by mimicking growth factor itself. Regulating the localization of growth factor in target tissue and concentration in therapeutic range and eliminating adverse side-responses are some of the long-term goals of growth factor delivery strategies. Therefore, further improvements can be obtained through dynamic stimuli-responsive scaffolds that efficiently promote desired cellular induction.

\section{Financial \& competing interests disclosure}

The authors have no relevant affiliations or financial involvement with any organization or entity with a financial interest in or financial conflict with the subject matter or materials discussed in the manuscript. This includes employment, consultancies, honoraria, stock ownership or options, expert testimony, grants or patents received or pending, or royalties. No writing assistance was utilized in the production of this manuscript.

\section{References}

1 Mercola M, Stiles CD. Growth factor superfamilies and mammalian embryogenesis. Development 102(3), 451-460 (1988).

2 Hynes RO. The extracellular matrix: not just pretty fibrils. Science 326(5957), 1216-1219 (2009).

3 Silva GA, Czeisler C, Niece KL et al. Selective differentiation of neural progenitor cells by high-epitope density nanofibers. Science 303(5662), 1352-1355 (2004).

4 Ustun S, Tombuloglu A, Kilinc M, Guler MO, Tekinay AB. Growth and differentiation of prechondrogenic cells on bioactive self-assembled peptide nanofibers. Biomacromolecules 14(1), 17-26 (2013).

5 Ceylan H, Tekinay AB, Guler MO. Selective adhesion and growth of vascular endothelial cells on bioactive peptide nanofiber functionalized stainless steel surface. Biomaterials 32(34), 8797-8805 (2011).
6 Kopesky PW, Vanderploeg EJ, Kisiday JD et al. Controlled delivery of transforming growth factor $\beta 1$ by self-assembling peptide hydrogels induces chondrogenesis of bone marrow stromal cells and modulates $S \operatorname{mad} 2 / 3$ signaling. Tissue Eng. Part A 17(1-2), 83-92 (2011).

7 Mammadov R, Mammadov B, Toksoz S et al. Heparin mimetic peptide nanofibers promote angiogenesis. Biomacromolecules 12(10), 3508-3519 (2011).

8 Mammadov R, Mammadov B, Guler MO, Tekinay AB. Growth factor binding on heparin mimetic peptide nanofibers growth factor binding on heparin mimetic peptide nanofibers. Biomacromolecules 13(10), 3311-3319 (2012).

9 Mammadov B, Mammadov R, Guler MO, Tekinay AB. Cooperative effect of heparan sulfate and laminin mimetic peptide nanofibers on the promotion of neurite outgrowth. Acta Biomaterialia 8(6), 2077-2086 (2012).

10 Rajangam K, Behanna HA, Hui MJ et al. Heparin binding nanostructures to promote growth of blood vessels. Nano Lett. 6(9), 2086-2090 (2006).

11 Chow LW, Bitton R, Webber MJ et al. A bioactive self-assembled membrane to promote angiogenesis. Biomaterials 32(6), 1574-1582 (2011).

12 Guo H, Cui G, Yang J et al. Sustained delivery of VEGF from designer self-assembling peptides improves cardiac function after myocardial infarction. Biochem. Biophys. Res. Commun. 424(1), 105-111 (2012).

13 Horii A, Wang X, Gelain F, Zhang S. Biological designer self-assembling peptide nanofiber scaffolds significantly enhance osteoblast proliferation, differentiation and 3-D migration. PLoS ONE 2(2), e190 (2007). 


\section{EDITORIAL | Tekinay, Guler, Mumcuoglu \& Ustun}

14 Guler MO, Soukasene S, Hulvat JF, Stupp SI. Presentation and recognition of biotin on nanofibers formed by branched peptide amphiphiles. Nano Lett. 5(2), 249-252 (2005).

15 Davis ME, Hsieh PCH, Takahashi T et al. Local myocardial insulin-like growth factor 1 (IGF-1) delivery with biotinylated peptide nanofibers improves cell therapy for myocardial infarction. Proc. Natl Acad. Sci. USA 103(21), 8155-8160 (2006).

16 Chen RR, Silva E a, Yuen WW, Mooney DJ. Spatio-temporal VEGF and PDGF delivery patterns blood vessel formation and maturation. Pharm. Res. 24(2), 258-264 (2007).

17 Giano MC, Pochan DJ, Schneider JP. Controlled biodegradation of selfassembling $\beta$-hairpin peptide hydrogels by proteolysis with matrix metalloproteinase-13. Biomaterials 32(27), 6471-6477 (2011).

18 Webber MJ, Newcomb CJ, Bitton R, Stupp SI. Switching of self-assembly in a peptide nanostructure with a specific enzyme. Soft Matter 7(20), 9665-9672 (2011).
19 Webber MJ, Tongers J, Newcomb CJ, Marquardt K, Bauersachs J. Supramolecular nanostructures that mimic VEGF as a strategy for ischemic tissue repair. Proc. Natl Acad. Sci. USA 108, 13438-13443 (2011).

20 Shah RN, Shah NA, Del MM et al. Supramolecular design of self-assembling nanofibers for cartilage regeneration. Proc. Natl Acad. Sci. USA 107, 3293 (2009). 